\title{
Green Manufacturing of Core-Shell Polyvinyl Alcohol-Gelatin Electrospun Nanofiber Scaffolds
}

\author{
Mustafa Şengör ${ }^{1}$, Mehmet Ali Gulgun ${ }^{2}$, Sabri Altıntaş ${ }^{1}$ \\ ${ }^{1}$ Dept. of Mechanical Engineering, Bogazici University \\ Bebek, 34342, Istanbul, Turkey \\ mustafa.sengor@boun.edu.tr; m-gulgun@sabanciuniv.edu \\ ${ }^{2}$ Department of Materials Science and Engineering, Sabanci University \\ Tuzla, 34956, Istanbul, Turkey \\ altintas@boun.edu.tr
}

\section{Extended Abstract}

In tissue engineering, natural/synthetic polymer based fibrous composite scaffolds obtained via electrospinning method were shown to support the cell adhesion and tissue regeneration. However, electrospinning of natural polymers requires the use of toxic solvents that are negatively affecting the cell proliferation and biocompatibility of the produced scaffolds in addition to the usage of acidic solvents which will result in massive biodegradation inside the body[1] .Here, a method was proposed that is higher safety for the patient and even for the experimentalists who are using harmful and highly volatile solvents.

Two types of polymers were used in the synthesis of the scaffolds by mimicking the key features of the tissue extracellular matrix which contains gelatin and coaxially organized nanofibers. Coaxial electrospinning technique was used to obtain core(PVA)-shell (gelatin) nanofibers. While material in the shell provides recognition sites for the tissue cells, core material provides mechanical endurance. Different from conventional methods, proposed work aims to lower the steps of application of the scaffold to the harmed tissue by using only deionized water as solvent. Instead of dissolving PVA and gelatin in toxic and acidic solvents, they were dissolved in the deionized water above the gelation temperature. Later, coaxial electrospinning generated increased cell spread and mechanical stiffness.

The samples were characterized by scanning and transmission electron microscopy. Based on the experimental results it is concluded that electrospun fibers obtained from the $8 \%$ concentrated gelatin solution had a beaded structure, whereas the coaxially fabricated PVA and gelatin from the same concentration solutions did not show any beaded morphology. Also core-shell fibers have diameters down to $180 \mathrm{~nm}$. This result showed that PVA aids to the uniform gelatin fiber formation which, may give higher mechanical stability. The electron microscopy analysis leading to these results has received support by the Nanotechnology Research and Application Center at Sabanci University.

\section{References}

[1] H.-C. Chen, W.-C. Jao, and M.-C. Yang, "Characterization of gelatin nanofibers electrospun using ethanol/formic acid/water as a solvent," Polym. Adv. Technol., vol. 20, no. 2, pp. 98-103, 2009. 